\title{
F-actin, tubulin and spectrin in the organ of Corti: Comparative distribution in different cell types and mammalian species ${ }^{1}$
}

\author{
Yehoash Raphael *, Brian D. Athey, Yu Wang, Michael K. Lee, Richard A. Altschuler \\ Kresge Hearing Research Institute. The Unirersity of Michigan, 1301 East Ann Street, Ann Arbor, MI 48109.0506. USA
}

(Received I September 1993; Revision received 2 February 1994; Accepted 14 February 1994)

\begin{abstract}
Laser scanning confocal microscopy was used to determine the distribution of actin, spectrin and tubulin in whole mounts of the organ of Corti of guinea pig, monkey, rat and chinchilla. Actin, spectrin and tubulin were localized in all cell types in the auditory epithelium. No specialized cytoskeletal organization of tubulin was detected in the cytoplasmic domain of hair cells. The only specialized organization of actin and spectrin in the cytoplasmic domain was the infra-cuticular network, found exclusively in apical guinea pig outer hair cells. In contrast, the lateral wall of inner and outer hair cells contained a homogenous distribution of label specific for actin and spectrin. The label intensity was similar in the base and the apex of the cochlea. These results indicate that the distribution of spectrin and actin in the auditory epithelium is similar to that in other epithelial cells, suggesting that actin and spectrin participate in the formation of cellular shape and possibly in docking molecules to the membrane.
\end{abstract}

Key words: Organ of Corti; Confocal microscopy; Cytoskeleton; Membrane skeleton; Histochemistry

\section{Introduction}

The cytoarchitecture of the organ of Corti and the shape and molecular organization of its cellular and extracellular elements are important factors in the function of the cochlea. The inner hair cells (IHCs) serve as its main mechanoelectrical transducers. The outer hair cells (OHCs) appear to play a major role in the active biomechanical mechanism that provides enhanced sensitivity and tuning in transduction (see Dallos, 1993, for review), perhaps through their motile response (Brownell et al., 1985; Kachar et al., 1986; Zenner, 1986). OHCs may actively exert forces during sound transduction. Previous studies of the cytoskeleton, membrane skeleton and plasma membrane of OHCs have provided a large amount of information on the cytoskeletal organization of the organ of Corti.

\footnotetext{
* Corresponding author. Fax: 313 764-0014; e-mail: Yehoash. Raphael $(a$ :Med.Umich.Edu.

'Presented, in part, in the Congress 'Progres en Otologie'. Bordeaux, December 1992, and at the 16ih Midwinter Meeting of the Association for Research in Otolaryngology, St. Petersburg, Florida. February 1993
}

Nevertheless, the cyto-molecular system and organization that underlie the active function of $\mathrm{OHCs}$ and its translation into cochlear mechanics have not been elucidated.

To understand the active and passive mechanics in the organ of Corti, it is important to determine the structural specialization of OHCs and supporting cells. From physical considerations of the size of most animal cells, it appears that surface tension is a dominant physical force between the lipid bilayer of the plasma membrane and the extracellular fluid (reviewed by Albrecht-Buehler, 1990). The theoretical and practical consequence of surface tension is that cells tend to be spherical shaped, unless a force greater than the surface tension is exerted, enabling cells to assume a shape other than round. Such forces may arise from internal cytoskeletal structures and/or by external adhesions with other cells or extracellular elements.

In the organ of Corti, both hair cells (HCs) and supporting cells are highly specialized in structure and function. OHCs are elongated and cylindrical in shape, pillar cells are long and slender in their middle segment and more bulky in the basal and apical portions, whereas Deiters cells have a cuboidal-like basal portion, from which a slender process projects apically at 
an oblique angle. To maintain these shapes, cells must have a specialized cytoskeletal organization, as in the conspicuous stress fibers in cultured fibroblasts or the extensive array of actin filaments along neural axons. Indeed, elongated supporting cells in the organ of Corti, such as Deiters cells and pillar cells, have an extensive network of microtubules and F-actin running parallel to their long axis. In contrast, a cytoplasmic cytoskeletal network that may establish the shape of $\mathrm{HCs}$, especially OHCs, has not been found. Microtubules in the cytoplasmic domain of OHCs are few and are not organized in a pattern that seems to support cell shape (Steyger et al., 1989). Cytokeratins, the intermediate filament proteins which are present in most epithelial cells and are thought to provide mechanical stability to these cells, were not found in OHCs (Raphael et al., 1987; Anniko et al., 1989; Bauwens et al., 1991; Raphael and Altschuler, 1991; Kuijpers et al., 1991, 1992). In the absence of a conspicuous cytoplasmic cytoskeleton, the shape of OHCs most probably depends on the membrane skeleton.

The membrane skeleton is a cytoskeletal domain closely associated with the plasma membrane of many cell types. In the absence of a cytoplasmic cytoskeletal network in HCs, the membrane skeleton may conceivably undertake functions related to the establishment and control of cell shape. Actin, spectrin (also known as fodrin) and other proteins have been localized to the membrane skeleton in erythrocytes and non-erythroid cells (see Burridge et al., 1982; Bretscher, 1991; Repasky and Gregorio, 1991, for reviews on membrane skeleton and its various proteins).

In OHCs, actin and spectrin have been localized to the area of the lateral membrane (Holley and Ashmore, 1988, 1990; Slepecky, 1989; Ylikoski et al., 1990, 1992; Holley et al., 1992; Slepecky and Ulfendahl, 1992; Nishida et al., 1993; Weaver et al., 1993). Furthermore, the circumferential lattice, a mesh of actin and spectrin similar to that found in the membrane skeleton of red blood cells, has been described in OHCs (Holley and Ashmore, 1988, 1990; Bannister et al., 1988; Forge, 1991). The circumferential lattice is anchored to the lateral membrane via the so-called pillars, which are $5-10 \mathrm{~nm}$ wide structures of unidentified protein, first described in the guinea pig (Flock et al., 1986) and the mole rat (Raphael and Wroblewski. 1986).

At present, it is not known whether the static shape in HCs is formed by the same molecular system responsible for the dynamic mechanical characteristics associated with $\mathrm{HC}$ motility and the active biomechanical mechanism. It is important to determine whether cytoskeletal proteins associated with the membrane and the membrane skeleton in HCs are directly related to motility, maintenance of cell shape or a combination thereof. In any case, the cstablishment of shape in HCs and supporting cells in the organ of Corti is probably a crucial factor in normal function and in exertion of the forces created by the active process.

The goal of these studies was therefore to localize the cytoskeletal and membrane skeletal proteins actin, spectrin and tubulin in the organ of Corti, using laser scanning confocal microscopy (LSCM) and to evaluate their distribution in the context of possible functions.

\section{Materials and Methods}

We use three structural domains to describe HCs: (a) the apical domain, including the stereocilia, apical junctions and cuticular plate, (b) the lateral wall, including the lateral plasma membrane and the associated circumferential mesh and subsurface cisternae (SSC) and (c) the cytoplasmic domain, including the remaining cytoplasm and organelles. The apical domain can be resolved well without the aid of LSCM, as evidenced by several high-resolution fluorescence microscopy studies (Steyger et al., 1989; Raphael and Altschuler, 1992). In contrast, structures located in basal areas, closer to the basement membrane of this epithelium, cannot be well resolved because of glare resulting from out-of-focus signals. The use of LSCM provides the increased resolution necessary for analysis of the deeper areas of the tissue, away from the surface facing the objective lens.

The animals included in this study were 10 pig-. mented guinea pigs and one each of the following: rat, monkey, chinchilla and mouse. The preparation of the tissue in this study did not involve dehydration nor other steps that are required for plastic embedding or

\footnotetext{
Fig. 1. Z-series taken from whole ount of the second turn of a guinea pig organ of Corti labeled with phalloidin. (a) Actin-specific label is in the stereocilia of OHCs (arrow) and in association with the adherens junction complex between HCs and supporting cells. (b) The distribution of actin in the adherens junction is asymmetric, with more intense label at the supporting cell side of the junction, especially the medial and lateral sides of the supporting cells (arrow). (c) The apical domain of inner pillar cells is heavily labeled with phalloidin. Intense label is also seen in the apical domain of the outer pillar cells (arrow). d. The cytoplasm of OHCs in all three rows $(1,2,3)$ lacks actin label, but a distinctive band of label is seen around the lateral membrane of the cells. (e) Actin is seen around the OHC perimeter and in the Deiters cell cup which is in contact with the basal domain of OHC. Label is found in the phalangeal processes of Deiters cells that traverse obliquely on their way to the luminal surface (arrow). (f) Intense actin label is in the basal domain of the outer pillar cells and in the Deiters cell cup. A faint and slender line of actin label is present in the perimeter of OHCs. Bar, $20 \mu \mathrm{m}$.
} 


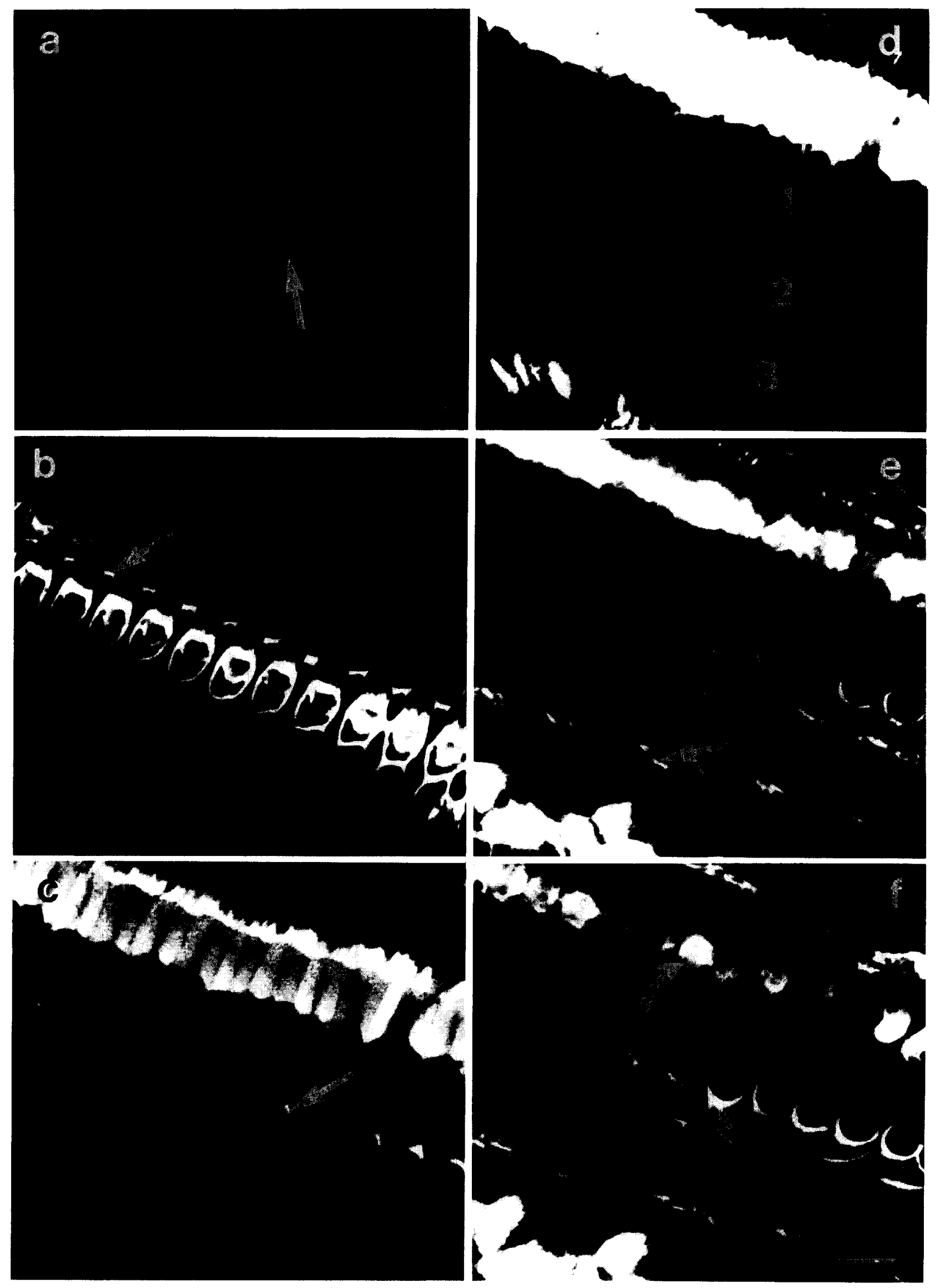


scanning electron microscopy, such as osmication or critical point drying. Therefore, shrinkage and other structural artifacts were minimized. Fixation was done either systemically, through intracardiac perfusion, or locally, via cochlear perfusion. For systemic perfusion, animals were deeply anesthetized and perfused with $2 \%$ paraformaldehyde in $0.15 \mathrm{M}$ phosphate buffer at $\mathrm{pH}$ 7.35. For local fixation, the bullae were removed from the skull, and the cochleae were rapidly exposed and immersed in a solution containing $2 \%$ paraformaldehyde, $0.15 \%$ Triton X-100 and rhodamine phalloidin (1:500, Molecular Probes) in phosphate buffer. This approach resulted in a very rapid fixation of the microfilament system. The primate inner ears were from an animal used for other projects and were shipped to our laboratory fixed in $4 \%$ paraformaldehyde.

The bony capsule surrounding the cochlea was removed with forceps. In the primate cochlea, the bony wall had to be thinned by drilling before it was possible to remove it with forceps. After fixation, the bony capsule was removed and the lateral wall tissues (spiral ligament and stria vascularis) were separated from the urgan of Corti, which remained attached to the modiolus. The process of immunolabeling was performed with the tissue in this state of dissection.

The tissue was further permeabilized with $0.3 \%$ Triton X-100 in PBS for $5 \mathrm{~min}$, incubated in primary antibody for $90 \mathrm{~min}$, rinsed thoroughly and incubated for 30 min with a solution containing fluorescein phalloidin and lissamine rhodamine-conjugated goat anti mouse secondary antibody $(1: 100$, Jackson ImmunoResearch, West Grove, PA). Primary antibodies were monoclonal anti-spectrin (Chemicon International, Temecula, CA) or monoclonal anti-tubulin (Sigma). All dilutions of immuno- and histochemicals were in PBS and all incubations were at room temperature. After a final rinse, the organ of Corti was separated from the modiolus and half turns were placed in the center of a microscope slide, between two spacing coverslips. A third coverslip was placed above the specimen. Several specimens were mounted directly between the glass slide and the coverslip, without spacers. The mounting solution was $60 \%$ glycerol in sodium carbonate buffer ( $\mathrm{pH} 8.5$ ) with $\mathrm{p}$-phenylenediamine to reduce fading.

Confocal $1.5 \mu \mathrm{m}$ optical sections were obtained using a Biorad MRC-600 LSCM attached to an inverted microscope (Nikon Diaphot). Images were obtained using X60 $(\mathrm{NA}=1.4)$ and $\mathrm{X} 100(\mathrm{NA}=1.4)$ oil objectives. Selected images were produced by scanning only a fraction of the field of view, effectively magnifying the original image file as much as eightfold. Image magnification was not modified by software-based image processing.

Full-screen double-label micrographs were obtained by taking a Z-series in the fluorescein channel using the BHS cube set, followed by successive rhodamine (lex $=547 \mathrm{~nm}$ ) micrographs using the GHS cube set. This was done because (a) it was found that, for double-label experiments, the cross-talk could be more effectively minimized using this method (see below), rather than by using the $\mathrm{K} 1 / \mathrm{K} 2$ cube set; and (b) the split-screen imaging option could be avoided, resulting in images with twice the field of view. In addition, no mechanical $X-Y$ translation of the specimen stage was performed between data collection runs. Post processing of the images using a cross-correlation subroutine (Inovision Corporation, Research Triangle Park, NC) indicated a minimal translational shift between the images, most likely due to chromatic shift (Athey, unpublished observations). Cross-talk between the channels was minimized by using very bright $0.1 \mathrm{~mm}$ green and red beads of appropriate wavelengths (Molecular Probes, Eugene, OR). The gain and the dark-current settings were adjusted to reject bead images from competing imaging channels. These markers were also used to verify the minimal $X-Y$ translation between imaging channels reported above. The vertical displacement between the two data sets along the $\mathrm{Z}$-axis was carefully monitored during data collection and found to be less than or equal to $0.5 \mu \mathrm{m}$. The $\mathrm{Z}$-axis motor is routinely calibrated, and backlash is corrected for in the Khoros software package (Bio-Rad, Cambridge, MA).

Micrographs were processed on a Sun Microsystems SPARC $_{2}$ workstation using the image analysis program

\footnotetext{
Fig. 2. A Z-series from the basal turn of the monkey cochlea $(\mathrm{a}-\mathrm{c})$, and single optical sections of the second turn of the guinea pig organ of Corti (d), and the middle portion (e) and the apical end ( $f$ ) of the monkey organ of Corti, labeled with fluorescent phalloidin. (a) In the apical domain. $\mathrm{OHC}$ actin label reveals three lines of stereocilia in a W-shaped bundle. Label in cuticular plate is in close proximity to the junctional actin, but actin-free gaps lie between the two actin domains. The fonticulus is a large actin-free space, surrounding the microtubule organizing center. Label in the adherens junction region includes both HC and supporting cell sides of the junction. (b) Several gaps are seen in the lower portion of the cuticular plate. (c) No ICN is observed in monkey; thus, subcuticular regions contain actin only in association with the lateral membrane of the cell. (d) Actin in the supporting cell side of the adherens junction is more abundant than in the HC side. The uppermost portion of the $1 C N$ is seen beneath the fonticulus (in second row OHCs). In more basal regions (third row OHCs), the ICN appears like a flower. Actin is evenly distributed at the perimeter of OHCs at this focal plane. (e) Intense actin labeling in the subapical region of pillar cells appears like a spring with the sharp tip directed laterally. Actin is distributed evenly around the perimeter of OHCs. Faint label is also detected in phalangeal processes of Deiters cells. (f) The spring-like actin in the pillar region is shorter in the apical turn. OHC perimeter and phalangeal processes of Deiters cells are distinctively labeled. Bars, $2 \mu \mathrm{m}$ in (c) and $10 \mu \mathrm{m}$ in (f) (for $\mathrm{d}-\mathrm{f}$ ).
} 


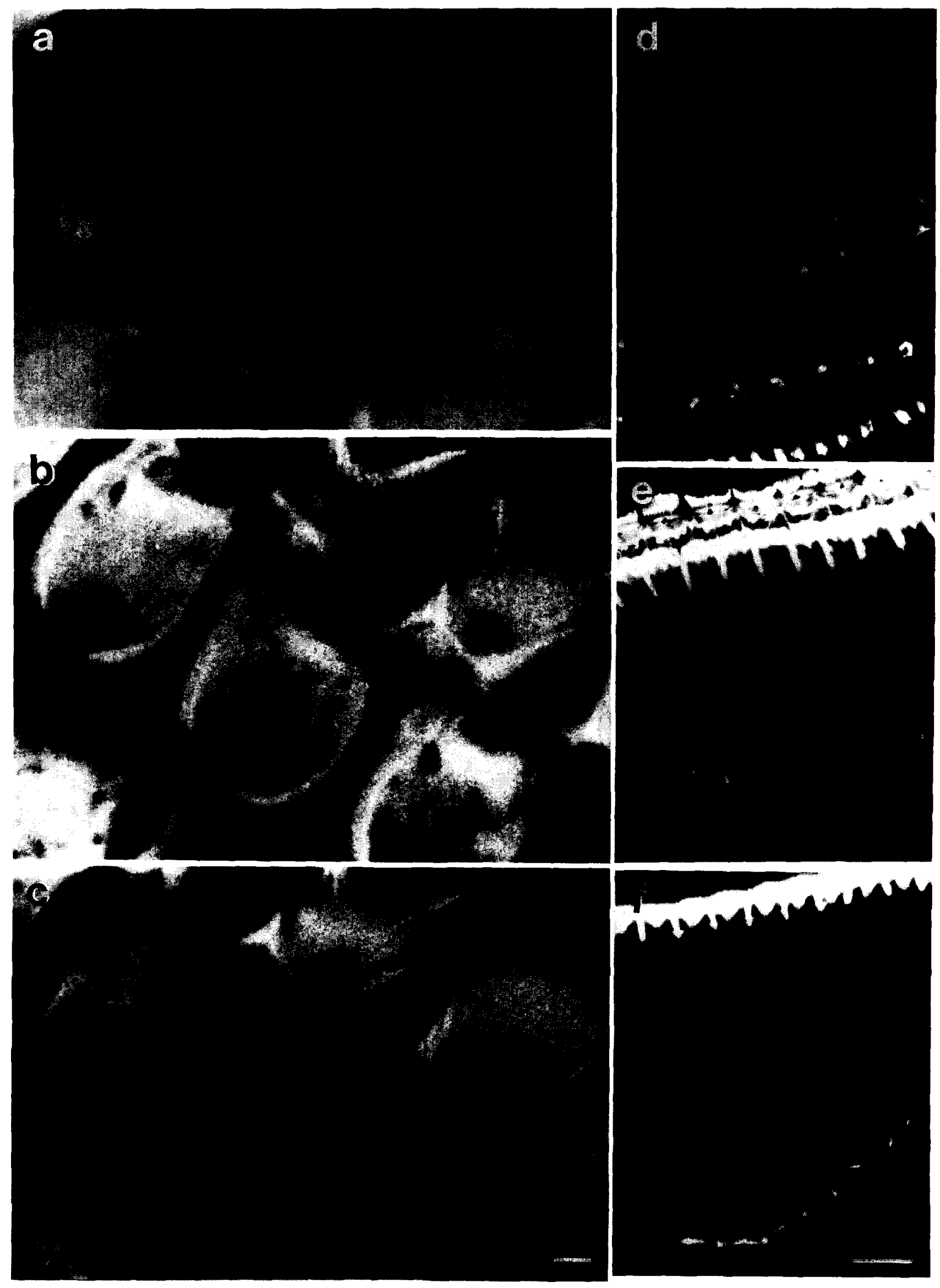


ANALYZE (Mayo Medical Ventures, Rochester, MN). Eight-bit raster files were produced and copied onto Kodak T-max 100 film with a Polaroid CI-5000 slidemaker.

\section{Results}

The distributions of actin, spectrin and tubulin were viewed in $\mathrm{z}$-axis series through the different levels of

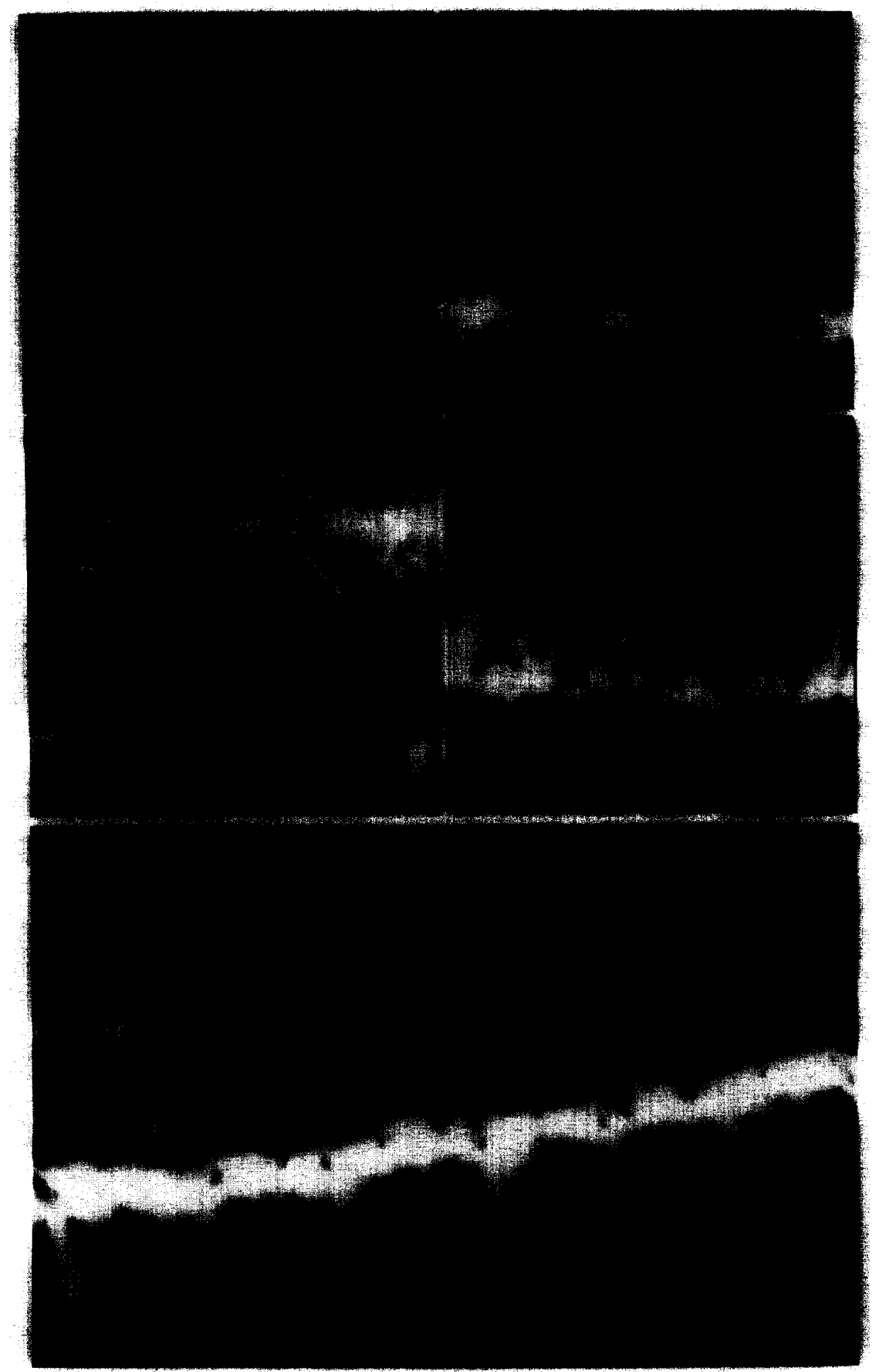


the organ of Corti, from reticular lamina to basilar membrane. Unless otherwise noted, results are common to all species investigated.

\section{Actin in $\mathrm{OHCs}$}

At low magnification, F-actin label is seen in the apical and the baso-lateral domain of OHCs in a Z-series of guinea pig organ of Corti (Fig. 1). At the apical domain of OHCs, labeled stereocilia show the W shape of the bundle (Fig. 1a). At the level of the adherens junction complex, the label is intense and the distribution on the supporting cell side of the junction is larger than on the $\mathrm{HC}$ side (Fig. 1b). Beneath the level of the cuticular plate and the adherens junction, the cytoplasm does not contain large amounts of actin (Fig. 1c-f).

The head plates of pillar cells label very intensely for actin (Fig. 1c-d). The label along the lateral membrane in the infra-junctional area is rather faint, yet clearly distinguishable around the cell perimeter and along the long axis (Fig. 1d-e). Actin is abundant in the Deiters cell cup, which half surrounds the basal domain of the $\mathrm{OHC}$ on its lateral aspect (i.e., away from the modiolus) (Fig. 1f). The cup is located at the lateral (away from the modiolus) side of the HC. The phalangeal processes of Deiters cells and the lower portion of the pillar cells also exhibit intense actin labeling. Analysis at low magnification, as shown in Fig. 1, allows us to assess the presence and condition of the various cellular components of the organ of Corti based on the distribution of known molecules.

Analysis at higher magnification permits a more detailed study of structure and molecular composition of the organ of Corti (Fig. 2). In the apical domain of OHCs, actin is known to be present in the stereocilia, cuticular plate and in association with the adherens junction. LSCM analysis of actin label in the apical domain of monkey OHCs reveals three rows of stereocilia extending apically from the apical surface (Fig. 2a). Individual stereocilia can be resolved. The cuticular plate extends to the lateral wall in some areas along the circumference of the cell, but in other areas, there are actin-free gaps separating the cuticular plate from the lateral wall. The single largest actin-free space is the fonticulus (Hawkins, 1976), the area where the basal body and the presumptive microtubule organizing center reside. Additional gaps are found in other areas along the perimeter of the OHCs (Figs. 2b, c). The gaps seem to be continuous through consecutive focal planes, suggesting that these gaps are actin-free gaps connecting the baso-lateral domain of $\mathrm{HCs}$ with the apical domain.

In the monkey organ of Corti, subcuticular regions contain actin only in association with the lateral membrane of the cell (Fig. 2c). This is also the case in the rat and the chinchilla (not shown). In contrast, OHCs in the guinea pig organ of Corti contain an infracuticular network (ICN) of actin (Held, 1926; Angelborg and Engström, 1973; Thorne et al., 1987). With the analytical power of the LSCM, it becomes apparent that the ICN is associated, at its apical end, with the fonticulus (Fig. 2d). From there, it descends into the central portion of the cell and appears in the optical sections like a flower with two or three petals (Fig. 2d). Thus, the predicted 3-D structure of the ICN is a multistranded core of actin. As previously reported (Thorne et al., 1987), the ICN is most prominent in the OHC of the third turn of the guinea pig cochlear duct.

Actin labeling at the subapical region of pillar cells is very intense. In the area where the head plates of inner and outer pillar cells overlap, an extension of actin is observed in the lateral direction (away from the modiolus), resembling a spring (Fig. 2e). Actin is prominent in association with the apical domain of OHCs, associated with stereocilia, the cuticular plate and the adherens junction, but it is also found in the perimeter, along the entire length of the lateral wall (Figs. 2e, f). The intensity of label appears similar in basal and apical turns of the cochlea. Actin is also found in the phalangeal processes of Deiters cells (Figs. 2e, f). In agreement with a previous report (Carlisle et al., 1988), an ICN of actin is not found in any of the mammals studied here, except for the guinea pig.

\section{Actin in IHCs}

The distribution of actin in the IHC area of the monkey inner ear is depicted in Fig. 3. Actin in IHCs is found in the stereocilia, cuticular plate and adherens junction. Stereocilia in monkey IHCs are arranged in two rows, one about twice as tall as the other (Fig. 3a). The area of contact between the cuticular plate and the adherens junction includes some gaps, similar to these observed in OHCs (Fig. 3b, compare to $2 \mathrm{a}$ and

Fig. 3. A Z-series (a-d) and a single optical section (e) showing the actin distribution in the IHC region of the monkey organ of Corti. (a) Stereocilia are arranged in two rows. (b) At the apical domain of IHCs, gaps can be seen between the cuticular plate and the functional actin ring. IHCs appear surrounded by four or five supporting cells. The latter have an apical contour resembling a figure eight (arrows point to two ends of one supporting cell). A faint line of staining is along the lateral membrane of the IHCs. (c) Supporting cells between IHCs contain large amounts of actin in the subapical region. Actin cables extend laterally in the area of overlap between the inner and outer pillar cells (curved arrow). (d) The actin in the apical domain of pillar cells appears like a spring (curved arrow). (e) An inage connused of several sections, depicting the spatial relationship between the spring (curved arrow) and other elements in the IHC region, including the IHC (ihc) and the inner pillar cell (ipc). Bars, $10 \mu \mathrm{m}$ in (d) and $5 \mu \mathrm{m}$ in (e). 
4e). IHCs are surrounded by 4 or 5 supporting cells. Two neighboring IHCs are separated by a supporting cell with a figure-eight shape (Fig. 3b). A faint but clear line of actin label is found along the contour of the lateral membrane of IHCs (Figs. $3 c$, d). Considering that no fluid space separates the lateral membrane of IHCs from neighboring supporting cells, it is not possible determine unequivocally whether the label is 
in the $\mathrm{HC}$, the supporting cell or both based on the present data.

Large amounts of actin are present in the apical domain of pillar cells (Figs. 3c, d). A spring-like structure made of actin is found in the pillar cell area (Fig. $3 \mathrm{~d})$. The spatial relationship between the spring and the other actin-containing structures in the IHC region of the apical cochlear turn is presented in Fig. 3e.

\section{Spectrin in the organ of Corti}

Spectrin immunoreactivity was detected in the cuticular plate and the lateral membrane of monkey OHCs, but not in the stereocilia (Fig. 4a). In the infra-cuticular area of guinea pig OHCs, spectrin was co-localized with the actin in the ICN and in the lateral membrane, immediately beneath the adherens junction (Fig. 4b). Heterologous junctional complexes between adjacent supporting cells also contained a faint spectrin-specific label (Fig. 4b). The lateral membrane label was evenly distributed along the long axis of the cell and along its round circumference (Fig. 4a). As the focal plane approached the basal domain of the HCs, label in the Deiters cup, and possibly in neural tissue, created asymmetry in the distribution of the label around the perimeter (Fig. 4c). The narrow phalangeal processes of pillar cells contained large amounts of spectrin, not only in the periphery but also in the central core of the cell (Fig. 4d). It therefore appeared that spectrin was co-localized with actin in all domains except the stereocilia.

\section{Actin and spectrin in the cuticular plate}

The distribution of actin and spectrin in the cuticular plate is similar in guinea pig and monkey preparations (guinea pig results are shown below). At the apical domain of IHCs, spectrin is found in the cuticular plate, which extends laterally to meet the junctional complex. The fonticulus does not show spectrin immunolabeling (Figs. 5a, b). In the infracuticular region, spectrin is associated with the lateral membrane (Figs. $5 b$, c). Spectrin-free areas are also present as gaps between the lateral membrane and the cuticular plate (Fig. 5b). A distinctive ring of spectrin is present along the adherens junction complex surrounding IHCs (Fig. $5 c)$. Spectrin is also found in junctional complexes between supporting cells located medial to the lHCs (Fig. 5c).
The cuticular plate of OHCs also shows positive immunolabeling for spectrin. The pattern of spectrinspecific label is not precisely co-localized with the actin-specific label. Rather, spectrin is packed into dense strands that seem to extend from the center of the cell to the periphery in a quasi-radial fashion (Fig. 5d). In contrast, actin in the cuticular plate is evenly distributed (Fig. 5f). Gaps are seen in the periphery of the cuticular plate, along the line of contact between the cuticular plate and the circumferential adherens junction with both spectrin (Fig. 5d) and actin (Fig. 5e) labels. The gaps were present at different optical sections (at the Z-axis) suggesting that cuticular-free channels run between the lateral membrane and the cuticular plate. The size of the gaps varies at different focal planes (compare Figs. 5d to 5e). The fonticulus contains neither spectrin nor actin (Figs. $5 \mathrm{~d}, \mathrm{e}$ ).

\section{Tubulin in the organ of Corti}

Tubulin immunoreactivity is present in all cells in the organ of Corti, although supporting cells reveal more tubulin-specific label than HCs (Fig. 6). In the apical domain of OHCs, tubulin staining is prominent in the fonticulus (Figs. 6a, b) and around the area of the cuticular plate, but the actin-rich area in the cuticular plate does not contain tubulin. Thus, the expressions of actin and tubulin are mutually exclusive in the apical domain of OHCs. In the infracuticular area there is a considerable amount of microtubule-specific label (Fig. 6c). The cytoplasm in the infranuclear domain of OHCs contains less microtubules than in the apical region (Fig. 6d). In the apical domain of IHCs, tubulin label is mostly peripheral, close to the lateral membrane of these cells (Figs. 6a, b). Supporting cells that surround IHCs contain very intense tubulinspecific labeling (Fig. 6a-c). Pillar cells have abundant microtubules in their apical plates (Fig. 6d) and in the narrow phalangeal processes (Fig. 6e). The apical processes of outer pillars and 3rd row Deiters cells also contain a large concentration of microtubules (Fig. 6a). At the level of the 'cup' which supports the basal part of OHCs, Deiters cells contain large amounts of microtubules. In the lower portions of Deiters cells, near the basilar membrane, microtubules are also abundant (Fig. 6f). The protein is organized in the perimeter of the cell, leaving a microtubule-free central core.

\footnotetext{
Fig. 4. LSCM sections showing the distribution of spectrin in the organ of Corti of monkey (a and $c$ ) and guinea pig (b and d). (a) Spectrin immunoreactivity is in the cuticular plate of third row OHCs and the lateral membrane of first and second OHCs. The lateral membrane label was evenly distributed along the long axis of the cell and along its round circumference. Spectrin label is found in the spring in the pillar region (arrow), in the junctions between pillar cells, and in the lateral membrane of lHCs (curved arrow). (b) Spectrin-specific label is found in the lateral perimeter of OHCs and in the ICN. Junctional complexes between supporting cells contain a faint spectrin-specific label. (c) Near the basal domain of $\mathrm{OHCs}$, label in the Deiters cup, and possibly in neural tissue, created increased staining, similar to the Deiters cup found with actin label (compare to Fig. 1f). (d) Intense spectrin-specific label seen in the phalangeal processes of pillar cells. $20 \mu \mathrm{m}$ in (a), $5 \mu \mathrm{m}$ in (b), 20 $\mu \mathrm{m}$ in (c) and $10 \mu \mathrm{m}$ in (d).
} 


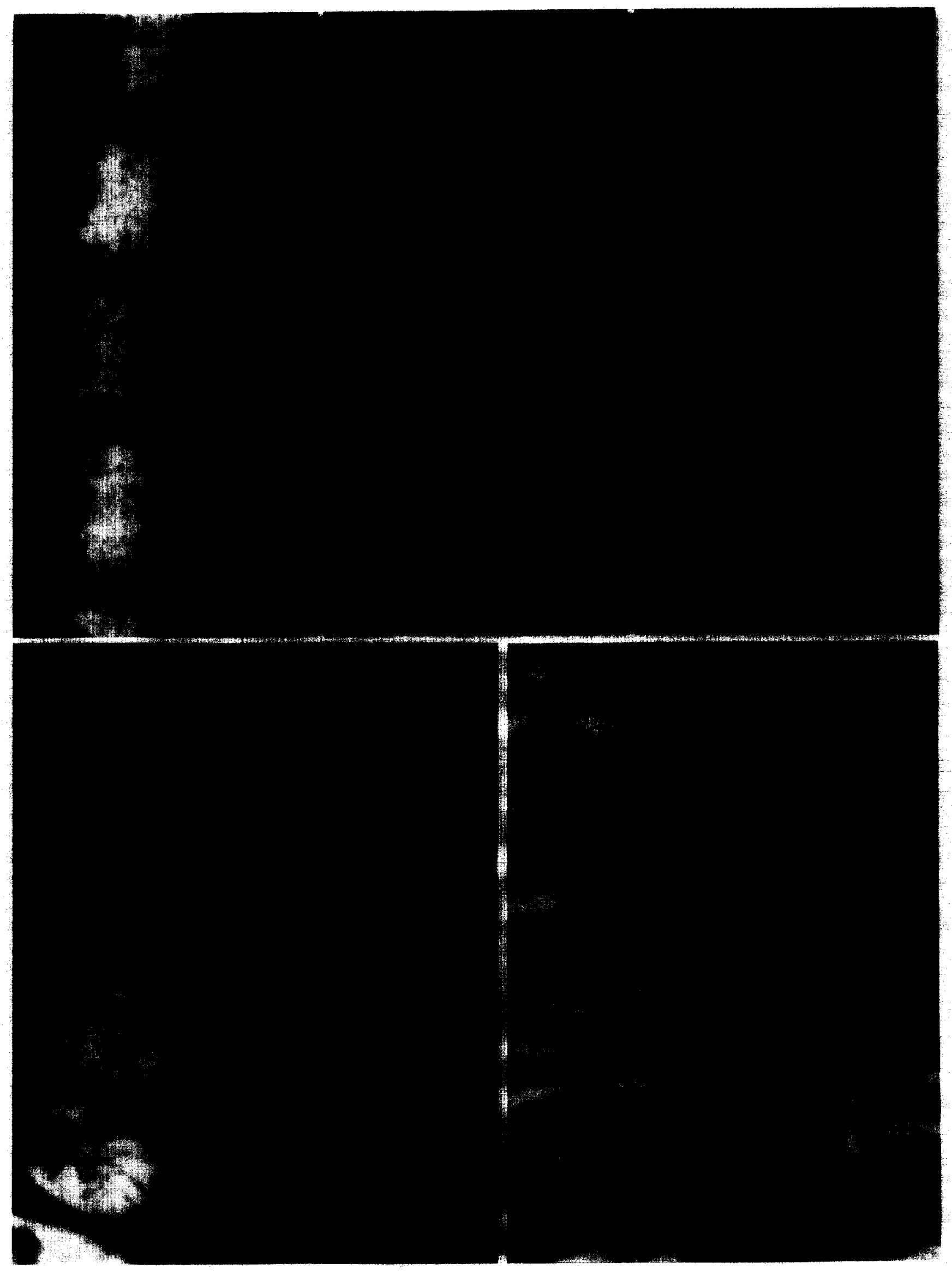


Nerve fibers that run longitudinally above the basilar membrane, parallel to the rows of Deiters cells, contain numerous microtubules. Branching sites of these nerves are recognized using microtubule-specific labeling (Figs. 6e, f).

\section{Discussion}

We used an improved fixation protocol for optimal preservation of the microfilament cytoskeleton and LSCM. With this method, subtle details of the distribution of spectrin and actin in HCs and supporting cells were observed. The results show that both actin and spectrin are present in the lateral wall of OHCs as well as supporting cells, and most probably IHCs. In OHCs, label specific for both actin and spectrin was visualized along the entire length of the lateral membrane. No major differences in the distribution of actin and spectrin in the lateral membrane were found between different turns or rows of the cochlear duct nor between cochleae of monkey, guinea pig, chinchilla, rat and mouse. Tubulin was detected in all cells in the organ of Corti, as previously reported (Steyger et al., 1989). The distributions of actin and tubulin in the apical domain of HCs were mutually exclusive.

The present results extend earlier reports on the presence of actin and spectrin along the lateral wall of OHCs in several respects: They show that both actin and spectrin are present all along the lateral wall. Actin and spectrin appear to be present along the borders of all $\mathrm{HCs}$ and supporting cells in the organ of Corti. Actin and spectrin are partly co-localized in the cuticular plate, but the distribution of spectrin is not completely homogeneous in this region. A spring-like organization of actin is found in pillar cells, at the area of overlap between the inner and outer pillars.

\section{Actin in the lateral wall}

Previous studies have reported the presence of actin in different structural domains of HCs (Flock and Cheung, 1977; Flock et al., 1982, 1986; Slepecky and Chamberlain, 1982; 1985; Zenner, 1986). Using immunochemistry at the electron microscope level, Flock et al. (1986) have demonstrated actin-specific antibody binding near the inner aspect of the plasma membrane of OHCs. With the advent of LSCM, Ylikoski et al.
(1992) used fluorescent phalloidin to label F-actin. They have demonstrated the presence of actin in the apical domain of HCs but failed to detect actin all the way down the lateral membrane, nor did they find much actin in the wall of OHCs in apical turns. In contrast, the present results demonstrate the presence of F-actin along the entire lateral membrane of $\mathrm{HCs}$ in all rows and turns.

\section{Spectrin in the lateral wall}

Spectrin has been localized to the lateral wall of OHCs by Holley and Ashmore (1988). Using light microscopy immunocytochemistry, they reported the presence of spectrin in association with the lateral OHC membrane. This finding was later confirmed by Ylikoski et al. (1990; 1992). They found spectrin along the lateral wall of OHCs in all but the more apical turns. In our studies, we observe spectrin immunostaining along the lateral wall of HCs in all rows and turns.

Using immuno-electron microscopy, Nishida et al. (1993) localized spectrin to the area between the plasma membrane and the SSC, whereas Weaver et al. (1993) detected actin in similar locations. These data further support the structural model proposed by Holley and Ashmore (1990), where circumferential filaments form a cortical lattice which is associated with other structures near the membrane of OHCs (reviewed by Dallos, 1993).

\section{Actin and spectrin in the ICN}

We detected actin and spectrin in the lateral membrane of cells in apical as well as basal areas of the cochlear duct in all mammals studied. The only conspicuous inter-species difference was the ICN, which was only found in the apical two turns of the guinea pig cochlea (Zenner, 1981; Thorne et al., 1987). Actin and spectrin were co-localized in the ICN of guinea pig OHCs, in agreement with previous studies (Holley and Ashmore, 1990; Ylikoski et al., 1992). The molecular composition of the ICN may indicate a structural similarity to the cuticular plate, but structural continuity with the cuticular plate was not observed. Rather, serial optical sectioning with the LSCM revealed that the ICN originated at its apical end from the area immediately beneath the fonticulus, that is, from the cuticular plate-free area, as previously reported by Ylikoski et al. (1992). The association of ICN with the

Fig. 5. Z-series through the guinea pig organ of Corti showing spectrin in IHCs (a-c) and single images of spectrin (d) and actin (e) labels in OHCs. (a) Intense spectrin label is found in the cuticular plate, and the fonticulus is unlabeled. (b) Spectrin-free gaps are seen between the lateral membrane and the cuticular plate. (c) In the infracuticular region, spectrin is associated with the adherens junction complex surrounding IHCs and in inter-supporting cell junctional complexes, medial to the IHCs. (d) Spectrin-specific label in the cuticular plate of OHCs is packed into dense strands, extending in a quasi-radial direction to the pcriphery of the cells. (e) Actin label in OHC cuticular plate is homogeneous, but actin-free gaps between the cuticular plate and the circumferential adherens junction form gaps through the periphery of the cuticular plate. The fonticulus contains neither spectrin nor actin. Bars, $5 \mu \mathrm{m}$ in (c) (for a-c), $5 \mu \mathrm{m}$ in (d) and $2 \mu \mathrm{m}$ in (e). 


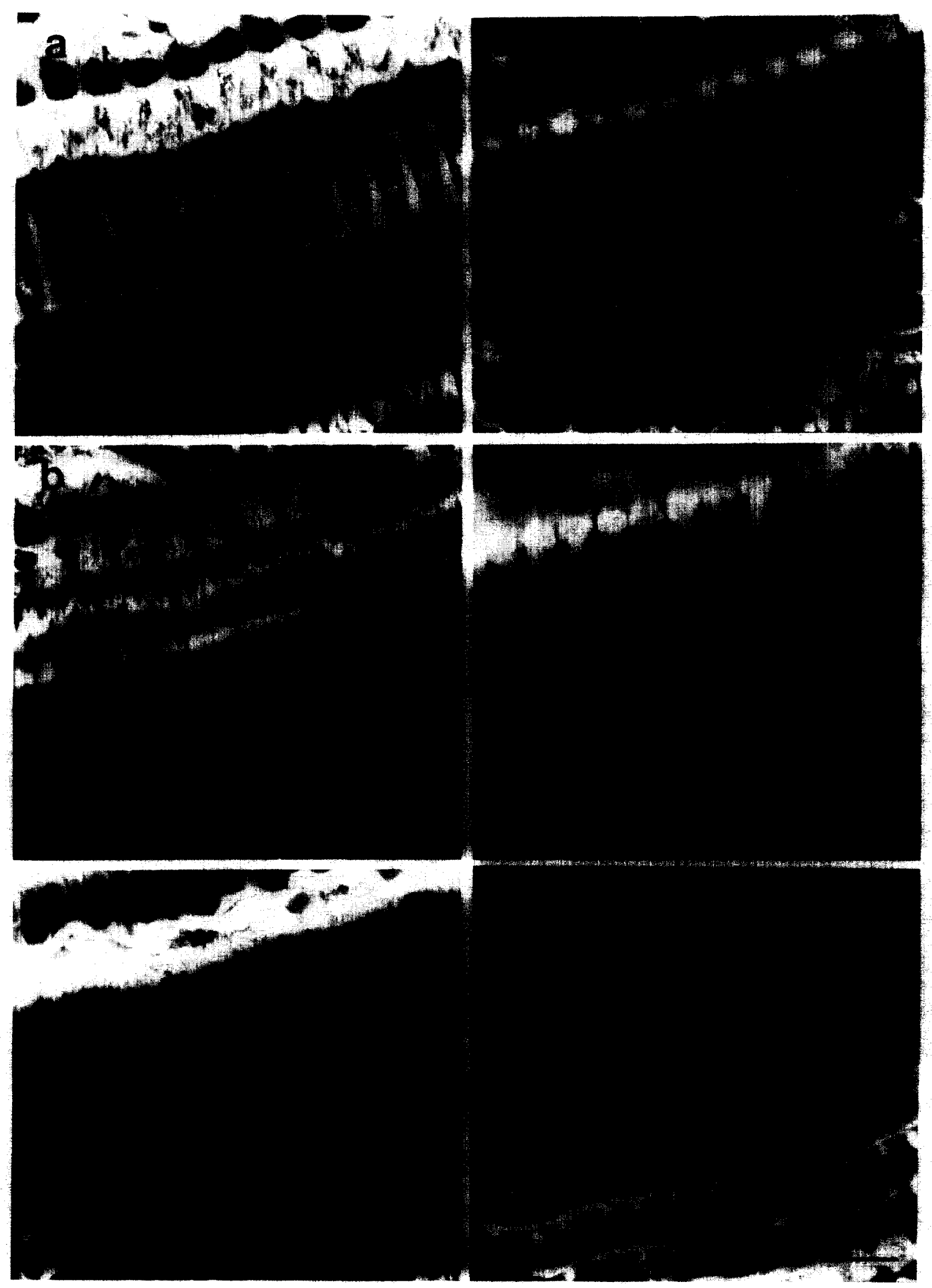


fonticulus may suggest a functional relation of the ICN to the basal body. The role of the basal body in mature $\mathrm{HCs}$ is not clear, but recent studies have shown that toxins are concentrated in the fonticulus (Hiel et al., 1992; El Barbary et al., 1993), raising the possibility that it is involved in detoxification in the OHC. Alternatively, the basal body is generally thought to be involved in the establishment of cellular orientation in space and polarity along the plane of the luminal surface (reviewed in Albrecht-Buehler, 1990). Future experiments are necessary to elucidate a possible role for the ICN in these functions.

\section{Precise localization of spectrin and actin}

At the light microscope level, it is not possible to resolve whether actin and spectrin in the lateral membrane of OHCs are associated with the SSC or with the membrane skeleton. The number of layers of SSC varies among different mammals ( 1 in human, monkey and rat and up to 10 in the guinea pig), yet the intensity of label did not seem to vary between these species. Moreover, in the guinea pig, the number of SSC layers is larger in the apical regions of the cochlea (our unpublished quantitative observations). Nevertheless, the label intensity for spectrin and actin appear similar in the apex and the base. Therefore, similarity in distribution of actin and spectrin between different mammals and between apical and basal regions of the cochlea can be taken as evidence that these molecules are not associated with the SSC. The data are therefore in agreement with previous reports that the mesh of spectrin is part of the membrane skeleton (Holley and Ashmore, 1990) and that spectrin is localized between the lateral plasma membrane and the SSC (Nishida et al., 1993).

\section{The role of actin and spectrin in the organ of Corti}

In the case of OHCs, it has been suggested that actin-spectrin membrane skeleton components are involved in active motile properties. Certainly, the presence of two potentially active and interacting molecules in the lateral wall of OHCs suggests a potential role in an active change of lateral wall length. Our results, however, do not provide evidence for their direct role in OHC motility. Rather, we found actin and spectrin in the lateral wall of OHCs and supporting cells, al- though only OHCs have a motile response. Moreover, the distribution of actin and spectrin in the lateral wall of OHCs appeared rather uniform along the cochlea duct, from base to apex. In contrast, base to apex gradients had been found in features such as the number of SSC (larger in apical turn, our unpublished data) or the number of efferent terminals on $\mathrm{OHCs}$ (Wright and Preston, 1973).

The distribution of actin and spectrin in OHC's is generally consistent with the role attributed to these molecules in most non-motile epithelial cells (see reviews in Bretscher, 1991; Repasky and Gregorio, 1991). The lattice mesh of actin and spectrin in HCs is similar to that found in the membrane skeleton of red blood cells and many epithelial cell types. We suggest that at least in HCs, which lack intermediate filaments (Raphael et al., 1987) and cytoskeletal actin filaments (Slepecky, 1989; Slepecky and Ulfendahl, 1992), the actin-spectrin mesh along the lateral wall is involved in the construction of actin networks for the establishment and regulation of cell shape. In addition, the circumferential mesh may participate in docking membrane units and anchoring cytoplasmic organelles to the lateral membrane. Such roles have been proposed previously (Slepecky, 1989; Holley and Ashmore, 1990).

While our results do not support a direct role for actin and spectrin in OHC motility, they certainly do not rule it out. It is possible that another molecule is selectively involved with modulating the role of actin and/or spectrin in OHC motility. It should also be considered that our localization of actin and spectrin in $\mathrm{OHCs}$ is consistent with an important indirect role in the motile response. Resistance and maintenance of shape are important to the transference of the motile response to cochlear mechanics, and as such, actin and spectrin play an important role in the mechanical features of the organ of Corti. Resistance to dynamic changes may be a role of supporting cells in the organ of Corti, which may require that they have a stable membrane skeleton. The spring of actin and the narrow phalangeal processes of pillar cells that contain large amounts of spectrin in the membranal and cytoplasmic domains may be important for transference of motility.

The present data reveal that at the level of the reticular lamina, two neighboring IHCs are separated

Fig. 6. A Z-series through the guinea pig organ of Corti labeled with tubulin-specific antibodies. (a) Tubulin-specific label in the apical domain OHCs is found in the fonticulus and along the perimeter of the cells, around the area of the cuticular plate. Intense label is found in all supporting cells, especially pillar cells and the apical processes of third row Deiters cells. (b) The area of II ICs and the supporting cells around them contains more tubulin than the OHC region. Tubulin is found around the periphery of IHCs and in the pillar cells. (c) In lower areas of OHCs, beneath the cuticular plate, higher tubulin-specific signal is seen. Supporting cells in the OHC region also label intensely. (d) The cytoplasm in the lower areas of OHCs contains little label for microtubules. Pillar cells display intense labeling. (e) The narrow phalangeal processes of pillar cells label intensely for tubulin. Deiters cells contain large amounts of microtubules in the 'cup' that surrounds and supports the basal part of OHCs. (f) The basal part of Deiters cells contains a large amount of microtubules, organized in the perimeter of the cell. leaving a microtubule-free central core. Nerve bundles which run near Deiters cells contain a large amount of microtubules. Bar. $20 \mu \mathrm{m}$. 
by a supporting cell with a figure-eight shape. This shape of supporting cells is similar to that of the phalangeal processes of Deiters cells in the $\mathrm{OHC}$ region. This may suggest a role for these cells in scar formation during IHC loss, similar to that shown during OHC degeneration after trauma (Raphael and Altschuler, 1991). The 3-D organization of supporting cells around IHCs needs to be better understood in the physiological and pathological states.

\section{Actin-free gaps around the cuticular plate}

The cuticular plate extends laterally to meet the junctional complex, but not throughout the perimeter of the cell, as described previously (Thorne et al., 1987). Kachar et al. (1993) have noted similar findings in guinea pig HCs using electron microscopy. They noted that uncoated vesicles that appeared like clathrin were associated with microtubules in the gaps between the cuticular plate and the lateral membrane and suggested that the actin-free areas in the cuticular plate serve as routes for vesicular transport. The presence of microtubules close to the lateral membrane at the apical domain of $\mathrm{HCs}$, observed by Steyger et al. (1989) and in the present study, is in agreement with the observations of Kachar et al. (1993).

\section{Acknowledgments}

We thank Dr. Joseph E. Hawkins, Jr. for comments on the results and the prescntation. Walter Meixner provided invaluable and expert help with confocal microscope imaging and data processing. We are pleased to acknowledge Swarna Manian, Richard Mast, Carlos Ricotti and Anat Sapan for excellent technical help. This work was supported by NIH Program Project Grant DC-00078.

\section{References}

Albrecht-Buehler, G. (1990) In defense of "nonmolecular" cell biology. Int. Rev. Cytol. 120, 191-241.

Angelborg, C. and Engström, H. (1973) The normal organ of Corti. In: A.R. Møller (Ed.), Basic mechanisms of Hearing, Academic Press Inc, New York, p. 157.

Anniko, M., Thornell, L.E., Raemaekers, F.C.S. and Stigbrand T. (1989) Cytokeratin diversity in epithelia of the human inner ear. Acta Otolaryngol. 180, 385-396.

Bannister, L.H., Dodson, H.C. Astbury, A.R. and Douek, E.E. (1988) The cortical lattice: a highly ordered system of subsurface filaments in guinea pig cochlear outer hair cells. Prog. Brain Res. $74,213-219$.

Bauwens, L.J.J.M., Veldman, J.E., Bouman, H., Ramaekers, F.C.S. and Huizing, E.H. (1991) Expression of intermediate filament proteins in the adult human cochlea Ann. Otol. Rhinol. Laryngol, $100,211-218$.

Bretscher, A. (1991) Microfilament structure and function in the cortical cytoskeleton. Annu. Rev. Cell Biol. 7, 337-374.

Brownell, W.E., Bader, C.R., Bertrand, D. and de Ribeaupierre, Y.
(1985) Evoked mechanical responses of isolated outer hair celk Science 227, 194-196.

Burridge, K., Kelly, T. and Mangeat, P. (1982) Nonerythrocyte spectrins: Actin-membrane attachment proteins occurring in many cell types. J. Cell Biol. 95, 478-486.

Carlisle, L., Zajic, G., Altschuler, R.A., Schacht, J, and Thome, P.R. (1988) Species differences in the distribution of infracuticular F-actin in outer hair cells of the cochlea. Hear. Res. 33, 201-206. Dallos, P. (1993) The active cochlea. J. Neurosci. 12, 4575-4585.

El Barbary, A., Altschuler, R.A. and Schacht, J, (1993) Glutathione $S$-transferase in the organ of Corti of the rat: enzymatic activity, subunit composition and immunohistochemical localization. Hear. Res. $71,80-90$

Flock, $\stackrel{\AA}{A}$ and Cheung, H.C. (1977) Actin filaments in sensory hairs of inner ear receptor cells. J. Cell Biol. 75, 339-343.

Flock, Å., Bretscher, A. and Weber, K. (1982) Immunohistochemical localization of several cytoskeletal proteins in inner ear sensory and supporting cells. Hear. Res. 7, 75-89.

Flock, A., Flock, B. and Ulfendahl, M. (1986) Mechanisms of movement in outer hair cells and a possible structural basis. Arch. Otorhinolaryngol. 243, 83-90.

Forge, A. (1991) Structural features of the lateral walls in mammalian cochlear outer hair cells. Cell Tiss. Res. 265, 473-483.

Hawkins, J.E. Jr. (1976) Drug ototoxicity. In: W. Keidel and W. Neff (Eds.), Handbook of Auditory Physiology, Vol. V/3, SpringerVerlag, Heidelberg, p. 723.

Held, H. (1926) Die Cochlea der Sauger under der Vogel, ihre Entwicklung und ihr Bau. In: A. Bethe (Ed.), Handbuch der normalen und pathologischen Physiologie. Sprínger, Berlin. Vol. II, pp. 467-534.

Hiel, H., Schamel, A., Frre, J.P., Hayashida, T., Dulon, D. and Aran, J.M. (1992) Cellular and subcellular localization of tritiated gentamicin in the guinea pig cochlea following combined treatment with ethacrynic acid. Hear. Res. 57, 157-165.

Holley, M.C. and Ashmore, J.F. (1988) A cytoskeletal spring in cochlear outer hair cells. Nature 335, 635-637.

Holley, M.C. and Ashmore, J.F. (1990) Spectrin, actin and the structure of the cortical lattice in mammalian cochlear outer hair cells. J. Cell Sci. 96, 283-291.

Holley, M.C., Kalinec, F. and Kachar, B. (1992) Structure of the cortical cytoskeleton in mammalian outer hair cells. J. Cell Sci. $102,569-580$.

Kachar, B., Brownell, W.E., Altschuler, R. and Fex, J. (1986) Electrokinetic shape changes of cochlear outer hair cells. Nature 322 , 365-368.

Kachar, B., Battaglia, A., Jaeger, R. and Fex, J. (1993) Vesicular traffic around the cuticular plate. Abstr. Assoe. Res. Otolaryngol. 455, St. Petersburg Beach, FL.

Kuijpers, W., Tonnaer, E.L., Peters, T.A. and Ramaekers, F.C. (1991) Expression of intermediate filament proteins in the mature inner ear of the rat and guinea pig. Hear. Res. 52, 133-46.

Kuijpers, W., Tonnaer, E.L., Peters, T.A. and Ramaekers, F.C. (1992) Developmentally-regulated coexpression of vimentin and cytokeratins in the rat inner ear. Hear. Res. 62, 1-10.

Nishida, Y., Fujimoto, T., Takagi, A., Honfo, I. and Ogawa, K. (1993) Fodrin is a constituent of the cortical lattice in outer hair cells of the guinea pig cochlea: immunocytochemical evidence. Hear. Res. 65, 274-280.

Raphael, Y. and Wroblewski, R. (1986) Linkage of sub-membranecisterns with the cytoskeleton and the plasma membrane in cochlear outer hair cells. J. Submicroscop. Cytol. 18, 731-737.

Raphael, Y., Marshak, G., Barash, A. and Geiger, B. (1987) Modulation of intermediate filament expression in the developing cochlear epithelium. Differentiation 35, 151-162.

Raphael, Y. and Altschuler, R.A. (1991) Reorganization of cytoskeletal and junctional proteins during cochlear hair cell degeneration. Cell Motil. Cytoskeleton 18, 215-227. 
Raphael, Y. and Altschuler, R.A. (1992) Early microfilament reorganization in injured auditory epithelia. Exp. Neurol. 115, 32-36.

Repasky, E.A. and Gregorio, C.C. (1991) Plasma membrane skeletons. In: P.L. Yeagle (Ed.), The structure of biological membranes, CRC Press, Boca Raton, FL, pp. 449-505.

Slepecky, N. (1989) Cytoplasmic actin and cochlear outer hair cell motility. Cell Tissue Res. 257, 69-75.

Slepecky, N. and Chamberlain, S.C. (1982) Distribution and polarity of actin in the sensory hair cells of the chinchilla cochlea. Cell Tissue Res. 224, 15-24.

Slepecky, N. and Chamberlain, S.C. (1985) Immunoelectron microscopic and immunofluorescent localization of cytoskeletal and muscle-like contractile proteins in inner ear sensory hair cells. Hear. Res. 20, 245-260.

Slepecky. N.B. and Ulfendahl, M. (1992) Actin-binding and microtubule-associated proteins in the organ of Corti. Hear. Res. 57. $201-215$.

Steyger, P.S., Furness, D.N., Hackney, C.M. and Richardson, G.P. (1989) Tubulin and microtubules in cochlear hair cells: Comparative immunocytochemistry and ultrastructure. Hear. Res. 42, 1-16.
Thorne, P.R., Carlisle, L., Zajic, G., Schacht, J. and Altschuler, R.A. (1987) Differences in the distribution of F-actin in outer hair cells along the organ of Corti. Hear. Res. 30, 253-266.

Weaver, S.P., Hoffpauir, J. and Schweitzer, L. (1993) Actin distribution along the lateral wall of gerbil outer hair cells. Brain Res. Bull. 31, 225-228.

Wright, C.G. and Preston, R.E. (1973) Degeneration and distribution of efferent nerve fibers in the guinea pig organ of Corti. A light and scanning electron microscopic study. Brain Res. 58, 37-59.

Ylikoski, J., Pirvola, U., Narvanen, O. and Virtanen, I. (1990) Nonerythroid spectrin (fodrin) is a prominent component of the cochlear hair cells. Hear. Res. 43, 199-2013.

Ylikoski, J., Pirvola, U. and Lehtonen, E. (1992) Distribution of F-actin and fodrin in the hair cells of the guinea pig cochlea as revealed by confocal fluorescence microscopy. Hear. Res. 60). $80-88$.

Zenner, H.P. (1981) Cytoskeletal and muscle-like elements in cochlear hair cells. Arch. Otorhinolaryngol. 230, 81-92.

Zenner, H.P. (1986) Motile responses in outer hair cells. Hear. Res. 22. $83-90$. 